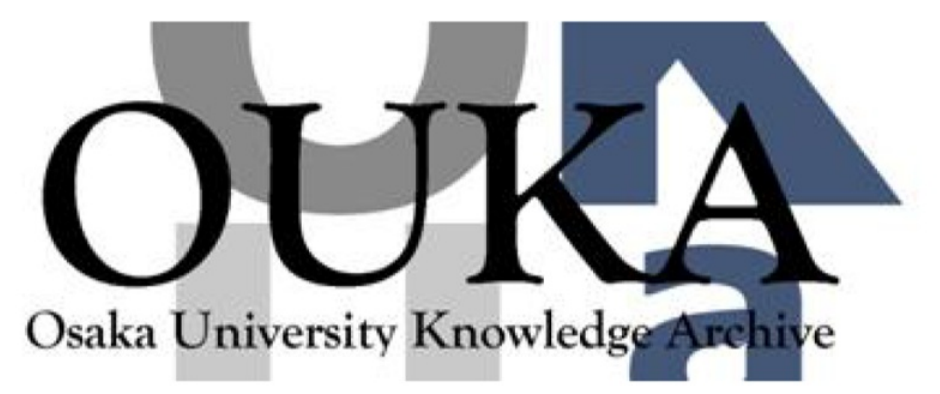

\begin{tabular}{|c|l|}
\hline Title & $\begin{array}{l}\text { Reactions and diffusion of atomic and molecular } \\
\text { oxygen in the Si02 network }\end{array}$ \\
\hline Author(s) & Tatsumura, K.; Shimura, T.; Mishima, E. et al. \\
\hline Citation & Physical Review B. 72(4) p. 045205 \\
\hline Issue Date & $2005-07-15$ \\
\hline oaire:version & VoR \\
\hline URL & https://hdl. handle. net/11094/85491 \\
\hline rights & $\begin{array}{l}\text { Copyright } 2005 \text { by the American Physical } \\
\text { Society. }\end{array}$ \\
\hline Note & \\
\hline
\end{tabular}

Osaka University Knowledge Archive : OUKA

https://ir. Library. osaka-u. ac. jp/

Osaka University 


\title{
Reactions and diffusion of atomic and molecular oxygen in the $\mathrm{SiO}_{2}$ network
}

\author{
K. Tatsumura, ${ }^{1, *}$ T. Shimura, ${ }^{2}$ E. Mishima,${ }^{2}$ K. Kawamura, ${ }^{2}$ D. Yamasaki,${ }^{1}$ H. Yamamoto, ${ }^{1}$ T. Watanabe,${ }^{1}$ \\ M. Umeno, ${ }^{3}$ and I. Ohdomari ${ }^{1,4}$ \\ ${ }^{1}$ Faculty of Science and Engineering, Waseda University, 3-4-1 Ohkubo, Shinjuku-ku, Tokyo 169-8555, Japan \\ ${ }^{2}$ Graduate School of Engineering, Osaka University, 2-1 Yamadaoka, Suita-shi, Osaka 565-0871, Japan \\ ${ }^{3}$ Faculty of Engineering, Fukui University of Technology, 3-6-1 Gakuen, Fukui-shi, Fukui 910-8505, Japan \\ ${ }^{4}$ Kagami Memorial Laboratory for Materials Science and Technology, Waseda University, 2-8-26 Nishi-waseda, Shinjuku-ku, \\ Tokyo 169-0051, Japan \\ (Received 22 February 2005; revised manuscript received 29 April 2005; published 13 July 2005)
}

\begin{abstract}
To address the reactions and diffusion of atomic and molecular oxygen in $\mathrm{SiO}_{2}$, the modification of the $\mathrm{SiO}_{2}$ network on exposure to an atomic or molecular oxygen atmosphere is investigated by measuring the $\mathrm{x}$-raydiffraction profile of the residual order peak emanating from the oxide. Analyses of the peak intensity and its fringe pattern provide experimental evidence for the recent theoretical predictions, indicating that atomic oxygen is incorporated into the $\mathrm{SiO}_{2}$ network near the surface and diffuses toward the interface along with modifying it even at a low temperature of $400{ }^{\circ} \mathrm{C}$, whereas molecular oxygen diffuses without reacting with the bulk $\mathrm{SiO}_{2}$ even at a temperature of $850{ }^{\circ} \mathrm{C}$ that is sufficiently high for oxidation reaction at the interface.

DOI: 10.1103/PhysRevB.72.045205

PACS number(s): 81.65.Mq, 66.30.Jt, 68.55.Jk, 61.10.Eq
\end{abstract}

\section{INTRODUCTION}

Silicon dioxide $\left(\mathrm{SiO}_{2}\right)$ films grown on crystal silicon $(\mathrm{Si})$ constitute the principal gate dielectrics in metal-oxidesemiconductor devices. With the continual shrinkage of device size, the atomistic understanding of all processes involved in silicon oxidation becomes increasingly important. ${ }^{1}$ In particular, the understanding of the diffusion of atomic and molecular oxygen in $\mathrm{SiO}_{2}$ is an outstanding problem, since oxidation kinetics is governed largely by the oxygen diffusion process. Although thermal oxidation of $\mathrm{Si}$ in a dry $\mathrm{O}_{2}$ atmosphere is one of the best methods of growing highquality $\mathrm{SiO}_{2}$, the growth rate is insufficiently low at low temperatures. In contrast with $\mathrm{O}_{2}$ molecules, atomic oxygen is expected to be more reactive. ${ }^{2}$ In fact, recent experiments using ultraviolet (UV) radiation ${ }^{3}$ or $\mathrm{Kr}$ electronic excitation $^{4-6}$ demonstrated the possibility of the rapid lowtemperature oxidation of $\mathrm{Si}$.

Growth kinetics of silicon oxidation using $\mathrm{O}_{2}$ molecules are reasonably well described by the Deal-Grove model, ${ }^{7}$ which postulates that molecular oxygen diffuses through the growing $\mathrm{SiO}_{2}$ layer and reacts at the $\mathrm{Si}-\mathrm{SiO}_{2}$ interface. ${ }^{18} \mathrm{O}$-tagged oxygen diffusion experiments show that there is no oxygen exchange in bulk $\mathrm{SiO}_{2}$ between oxygen molecules and network oxygen atoms. ${ }^{8,9}$ This result means that $\mathrm{O}_{2}$ diffuses in molecular form without reacting with a $\mathrm{SiO}_{2}$ network. This picture is also supported by the detection of interstitial oxygen molecules, ${ }^{10}$ by the linear dependence of the diffusion on the partial pressure of the gaseous $\mathrm{O}_{2}$ both in thermal oxides ${ }^{7}$ and silica membranes, ${ }^{8,11}$ and by the similar diffusion properties of argon (an atom of approximately the same size as $\mathrm{O}_{2}$ ). ${ }^{12}$ Furthermore, there is a consensus among recent theoretical investigations that molecular $\mathrm{O}_{2}$ in the spin-triplet state is energetically the most stable state in $\mathrm{SiO}_{2} \cdot{ }^{13-15}$

A diffusion process for atomic oxygen has been proposed by recent theoretical investigations. ${ }^{16-18}$ The diffusion pro- cess proposed is as follows: An oxygen atom inside a $\mathrm{SiO}_{2}$ tends to react with a $\mathrm{SiO}_{2}$ network and to form a stable $\mathrm{Si}-\mathrm{O}-\mathrm{O}-\mathrm{Si}$ structure (peroxy linkage). Diffusion of the introduced $\mathrm{O}$ atom consists of two steps: (i) a jump of the diffusing $\mathrm{O}$ atom around an adjacent $\mathrm{Si}$ and an incorporation of the atom into a neighboring $\mathrm{Si}-\mathrm{O}$ bond, via a transition state with a fivefold coordinated $\mathrm{Si}$ atom and a onefold coordinated $\mathrm{O}$ atom, and (ii) an exchange of the diffusing $\mathrm{O}$ atom and the network $\mathrm{O}$ atom via a transition state with onefold and threefold coordinated $\mathrm{O}$ atoms. Hence, it is considered that there are significant differences between the diffusion mechanisms of atomic and molecular oxygen. However, experimental evidence on the atomic oxygen diffusion has been lacking.

The purpose of this paper is to provide experimental evidence for the difference in reactions and diffusion of atomic and molecular oxygen in $\mathrm{SiO}_{2}$. Since overcoordinated and undercoordinated atoms are considered to promote switching of bond connections, ${ }^{19}$ the diffusion process of atomic oxygen, as well as their incorporation into the $\mathrm{SiO}_{2}$ network, may induce modifications of the $\mathrm{SiO}_{2}$ network. On the other hand, diffusion of $\mathrm{O}_{2}$ molecules is generally believed to proceed with no modification of the $\mathrm{SiO}_{2}$ network. In this work, we investigate whether modification of the $\mathrm{SiO}_{2}$ network occurs upon exposure to an atomic or molecular oxygen atmosphere by measuring the intensity profile of an $\mathrm{x}$-raydiffraction peak ${ }^{20-22}$ that reflects the average structure of an oxide film. The origin of the diffraction peak on which we focus has very recently been clarified to be the residual order of $\mathrm{Si}$ atoms within $\mathrm{SiO}_{2} \cdot{ }^{23,24}$ A silicon lattice is expanded during oxidation without completely disordering it. The intensity profile of the residual order peak can be used as a sensitive indicator for monitoring the structural changes of the thermal oxide. In fact, it was demonstrated that the peak intensity is decreased and ultimately removed by thermal annealing, indicating the decrease of residual ordering and complete conversion to amorphous $\mathrm{SiO}_{2} \cdot{ }^{25}$ 


\section{EXPERIMENT}

We compare x-ray-diffraction profiles for three samples that were reoxidized in an atomic or molecular atmosphere after formation of base $\mathrm{SiO}_{2}$ layers in a $100 \%$ dry $\mathrm{O}_{2}$ atmosphere. The substrates were boron-doped Cz-Si(001) wafers with resistivity of $10-15 \Omega \mathrm{cm}$ and an off-angle of $0.4^{\circ}$ toward the [010] direction. The base $\mathrm{SiO}_{2}$ layers of 24.7-nm thickness (by ellipsometry with a refractive index of 1.46) were obtained by dry $\mathrm{O}_{2}$ oxidation at $850{ }^{\circ} \mathrm{C}$ for $260 \mathrm{~min}$. The atomic oxygen-exposed sample, hereafter referred to as the $\mathrm{O}-400{ }^{\circ} \mathrm{C}$ sample, was obtained by reoxidizing the base $\mathrm{SiO}_{2}$ layer in an atomic radical $\mathrm{O}$ flow generated from high-density low-electron-temperature $\mathrm{Ar} / \mathrm{O}_{2}$ $(=2000 / 10 \mathrm{sccm}, 6.7 \mathrm{~Pa})$ plasma at a substrate temperature of $400{ }^{\circ} \mathrm{C}$ for $125 \mathrm{~min}$. The reoxidization process increased the oxide thickness by $1.5 \mathrm{~nm}$. The applied microwave power and frequency were $2500 \mathrm{~W}$ and $2.45 \mathrm{GHz}$, respectively. The plasma apparatus employed (Tokyo Electron, Trias SPA) was designed to enable damage-free processes. ${ }^{5,6,26,27}$ Two kinds of molecular oxygen-exposed samples were prepared as follows. One, hereafter the $\mathrm{O}_{2}$ $-400{ }^{\circ} \mathrm{C}$ sample, was prepared under the same conditions as those of the $\mathrm{O}-400{ }^{\circ} \mathrm{C}$ sample except for a microwave power of $0 \mathrm{~W}$; that is, the sample was exposed to a molecular $\mathrm{O}_{2}$ flow. The treatment did not increase the oxide thickness $(<$ $+0.1 \mathrm{~nm})$. The other, hereafter the $\mathrm{O}_{2}-850{ }^{\circ} \mathrm{C}$ sample, was reoxidized in a $100 \%$ dry $\mathrm{O}_{2}$ atmosphere at $850{ }^{\circ} \mathrm{C}$ until the thickness increase of $2.0 \mathrm{~nm}$ was achieved ( $25 \mathrm{~min})$. X-raydiffraction measurements using synchrotron radiation were performed on BL4C of the Photon Factory (KEK) by employing a four-circle diffractometer with a $\mathrm{Si}(111)$ crystal analyzer. The wavelength was chosen to be $0.154 \mathrm{~nm}$.

\section{RESULTS AND DISCUSSION}

Figure 1 shows the diffraction intensity profiles for the O-400, $\mathrm{O}_{2}-400$, and $\mathrm{O}_{2}-850{ }^{\circ} \mathrm{C}$ samples. In Fig. 1, the data for the sample with the base $\mathrm{SiO}_{2}$ layer not subjected to reoxidization, hereafter the base $\mathrm{SiO}_{2}$ sample, is shown. The intensity profiles are scanned along the crystal truncation rod (CTR) scattering extending from the 111 bulk Bragg peak. The CTR scattering is caused by the termination of Si lattice at the $\mathrm{Si}-\mathrm{SiO}_{2}$ interface and extends in the surface-normal direction, in this case, the direction rotated by $0.4^{\circ}$ with respect to [001] toward [010]. In Fig. 1, the 111 bulk peak is located at $l=1.0$ and the CTR scattering is observed as the intensity decreasing with distance $(d)$ from 111 according to $\sim 1 / d^{2}$.

For the base $\mathrm{SiO}_{2}$ sample, the residual order peak is clearly observed on CTR at $l=0.45$, corresponding to the position of $h, k, l=1.00,1.00,0.45$, in accordance with the previous reports. ${ }^{20-22}$ One of the characteristic features of the peak is that its profile exhibits a Laue-function-like oscillation-fringe pattern. As shown in Fig. 2, the enlargement of Fig. 1, the peak profile consists of a main peak and several subpeaks, and the width of the main peak is twice as much as those of the subpeaks. Furthermore, the oscillation period $(\lambda)$ is related to the inverse of the film thickness $(t)$ by

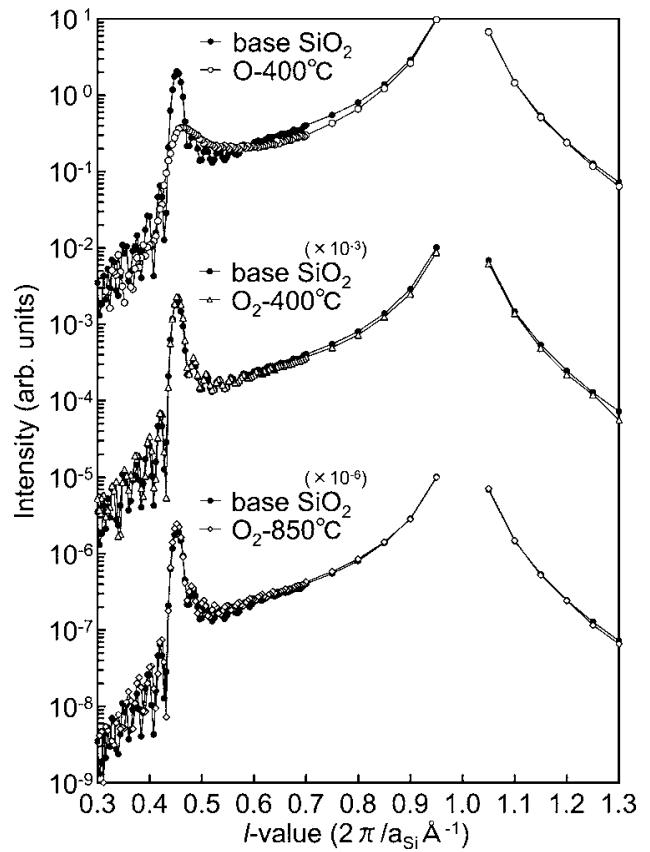

FIG. 1. X-ray-diffraction intensity profiles for the $\mathrm{O}-400{ }^{\circ} \mathrm{C}$ (circles), $\mathrm{O}_{2}-400{ }^{\circ} \mathrm{C}$ (triangles), and $\mathrm{O}_{2}-850{ }^{\circ} \mathrm{C}$ (squares) samples, coupled with the data for the base $\mathrm{SiO}_{2}$ sample (filled circles). The intensity profiles are scanned along the CTR scattering extending from the 111 bulk Bragg peak and are plotted as a function of the $l$ value in a silicon reciprocal lattice unit. The solid lines are guides for the eyes.

$\lambda=a_{\mathrm{Si}} / t$, where $a_{\mathrm{Si}}$ is the silicon unit-cell dimension. In this case, $\lambda$ is 0.023 , agreeing well with the value estimated from the thickness of the base $\mathrm{SiO}_{2}$ layer $(0.543 / 24.7=0.022)$.

Exposure of the base $\mathrm{SiO}_{2}$ layer to an atomic oxygen flow induces significant changes in its $\mathrm{x}$-ray-diffraction property. The residual order peak is observed also for the $\mathrm{O}-400{ }^{\circ} \mathrm{C}$ sample, but its intensity is approximately one order of magnitude smaller than that for the base $\mathrm{SiO}_{2}$ sample. In addition, the oscillation-fringe pattern around the peak completely disappears (Fig. 2). Note that the remarkable

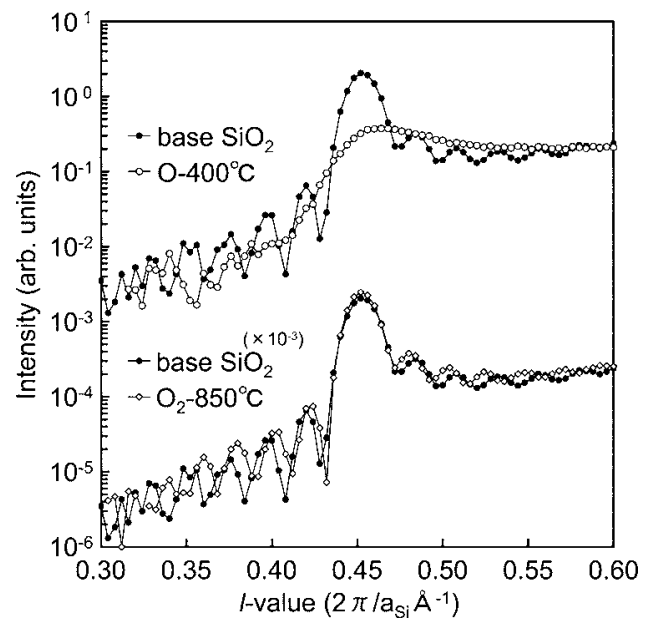

FIG. 2. Enlargement of the data in Fig. 1 for the $\mathrm{O}-400{ }^{\circ} \mathrm{C}$ (circles) and $\mathrm{O}_{2}-850{ }^{\circ} \mathrm{C}$ (squares) samples. 
coincidence of the data at the higher angle side of 111 ( $l$ $>1.0)$ in Fig. 1 allows us to compare the data between the samples at the lower angle side. In contrast to the case of the $\mathrm{O}-400{ }^{\circ} \mathrm{C}$ sample, the intensity profile for the $\mathrm{O}_{2}-400{ }^{\circ} \mathrm{C}$ sample does not differ from that of the base $\mathrm{SiO}_{2}$ sample (Fig. 1). Similarly, the intensity profile for the $\mathrm{O}_{2}-850{ }^{\circ} \mathrm{C}$ sample is almost the same as that for the base $\mathrm{SiO}_{2}$ sample, but the peak intensity becomes slightly larger and the period of oscillation slightly decreases, becoming 0.021 (Fig. 2). This change of the period precisely reflects the thickness increase of $2.0 \mathrm{~nm}$ (i.e., $0.543 / 26.7=0.020$ ). This change of the period together with the slight increase of the peak intensity indicates that the reoxidization process produced the oxide with the same structure as the base $\mathrm{SiO}_{2}$ at the $\mathrm{Si}-\mathrm{SiO}_{2}$ interface.

The decrease in the peak intensity at $l=0.45$ means an increase in the static disorder of $\mathrm{Si}$ atoms within bulk $\mathrm{SiO}_{2}{ }^{25}$ Hence, the result for the $\mathrm{O}-400{ }^{\circ} \mathrm{C}$ sample is solid evidence that atomic oxygen reacts with the bulk $\mathrm{SiO}_{2}$ and modifies its network structure even at a low temperature of $400{ }^{\circ} \mathrm{C}$. In contrast, the result for the $\mathrm{O}_{2}-400{ }^{\circ} \mathrm{C}$ sample indicates no structural change occurs during the exposure to the molecular oxygen flow at $400{ }^{\circ} \mathrm{C}$. For the $\mathrm{O}_{2}-850{ }^{\circ} \mathrm{C}$ sample, the thickness increase of $2.0 \mathrm{~nm}$ confirms that oxidant species needed to oxidize 6 or $7 \mathrm{Si}$ layers have reached the $\mathrm{Si}-\mathrm{SiO}_{2}$ interface. Despite the passage of such a considerable amount of oxidant species, the peak intensity does not decrease. This clearly shows that molecular oxygen diffuses without reacting with the bulk $\mathrm{SiO}_{2}$ even at a sufficiently high temperature of $850^{\circ} \mathrm{C}$ for oxidation reaction at the interface.

The shape of the peak profile provides further information about the residual order distribution. A clear oscillationfringe pattern around a diffraction peak is observed only for homogeneously ordered thin films having finite thickness and two distinct boundaries. If the structure is gradually disordered and converted to be amorphous near one of the boundaries, it is impossible to observe the oscillation pattern whose period is related to the film thickness.

To elucidate the structural change of the $\mathrm{O}-400{ }^{\circ} \mathrm{C}$ sample, we made two types of model structures and calculated the x-ray-diffraction profiles from them. Figure 3 schematically shows the positions of $\mathrm{Si}$ atoms in thermally grown ${ }^{23,24} \mathrm{SiO}_{2}$ and explains the concept of the residual order. In Fig. 3, it is supposed that the $\mathrm{Si}$ atoms are moved from their crystal positions in the surface-normal direction and disordered during oxidation, but the $\mathrm{Si}$ atoms still remain sufficiently ordered to produce a detectable diffraction peak. Recent molecular dynamics simulation demonstrated the possible existence of a $\mathrm{SiO}_{2}$ structure consisting of cornersharing $\mathrm{SiO}_{4}$ tetrahedrons with the bond length and angle of amorphous $\mathrm{SiO}_{2}$ and showing an average arrangement of $\mathrm{Si}$ atoms corresponding to the expanded Si lattice. ${ }^{23}$ Model A in Fig. 3(a) is a homogeneous structure in the sense that the static disorder for the $\mathrm{Si}$ atoms from the ordered positions is constant throughout the film. On the other hand, model B in Fig. 3(b) is a gradually disordered structure with a gradually increasing static disorder from the interface to the surface.

Figure 4 shows the $\mathrm{x}$-ray-diffraction profiles calculated for models A and B. The structures used for the calculation in Fig. 4 are shown in Fig. 5. The preparation procedure of the

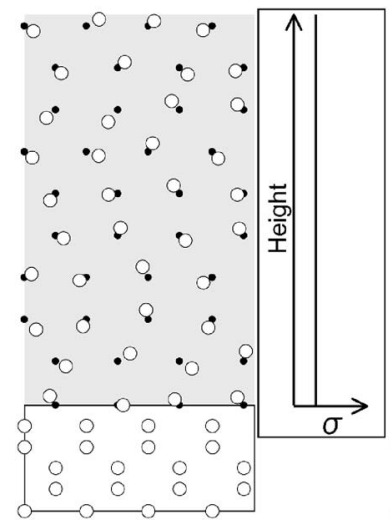

(a) Model A

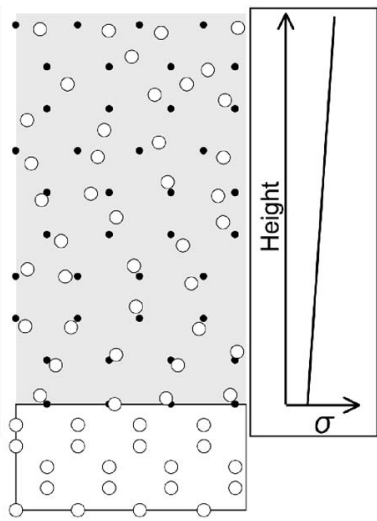

(b) Model B
FIG. 3. Schematic illustration of the positions of $\mathrm{Si}$ atoms in thermally grown $\mathrm{SiO}_{2}$. The open circles represent $\mathrm{Si}$ atoms. The inside of the box is the substrate region. The small black dots show the positions of the expanded Si lattice in the absence of static disorder: (a) Model A: a homogeneous structure and (b) Model B: a gradually disordered structure.

model structures is as follows. The (001)-terminated $\mathrm{Si}$ model (19 800 atoms) is formed, which is $11.93 \mathrm{~nm}$ thick along [001] (88 atomic layers) and $5.76 \mathrm{~nm}$ long along [1 $\overline{1} 0]$ and [110] $(15 \times 15$ atoms per atomic layer). The Si lattice, except for the substrate region (8 atomic layers), is expanded toward [001] with expansion ratio of 2.21, resulting in the expanded Si lattice of the thickness of $24 \mathrm{~nm}$. Each Si atom is displaced from its ordered position. Each component of the displacement vector, $\mathbf{d}(x, y, z)$, is generated according to the normal distribution function with a standard deviation $(\sigma), f(x)=(1 / \sqrt{2 \pi} \sigma) \exp \left(-x^{2} / 2 \sigma^{2}\right)$. Model A has a constant $\sigma$ of $0.8 \AA$. Model B has a linearly increasing $\sigma(z)$ with $\sigma$ $=0.8 \AA$ at the interface and $\sigma=2.2 \AA$ at the surface.

Despite their simplicity, models A and B, respectively, are able to reproduce the characteristic features of the residual order peaks for the base $\mathrm{SiO}_{2}$ and $\mathrm{O}-400{ }^{\circ} \mathrm{C}$ samples. The gradually disordered structure for the $\mathrm{O}-400{ }^{\circ} \mathrm{C}$ sample provides further evidence that atomic oxygen is incorporated into the $\mathrm{Si}-\mathrm{O}$ network near the surface and diffuses toward the interface along with modifying it. Note that the original structure of the thermal oxide is not completely homogeneous but shows an inclined distribution of the residual order

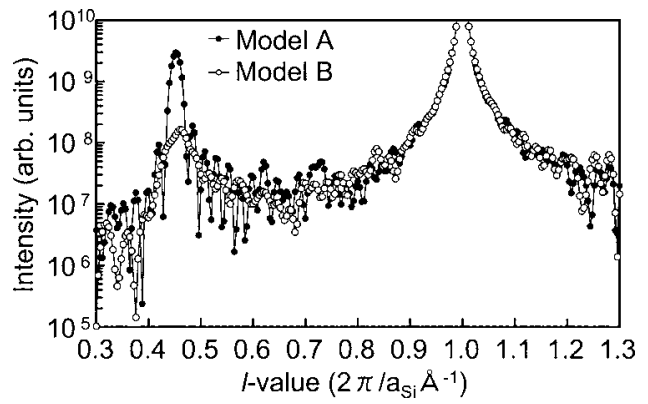

FIG. 4. X-ray-diffraction intensity profiles calculated for Model A and Model B in Fig. 5 on the basis of kinematical diffraction theory. 


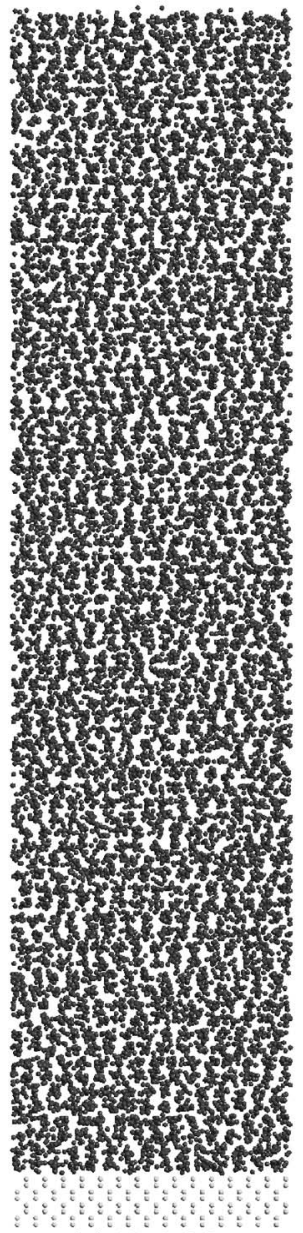

(a) Model A

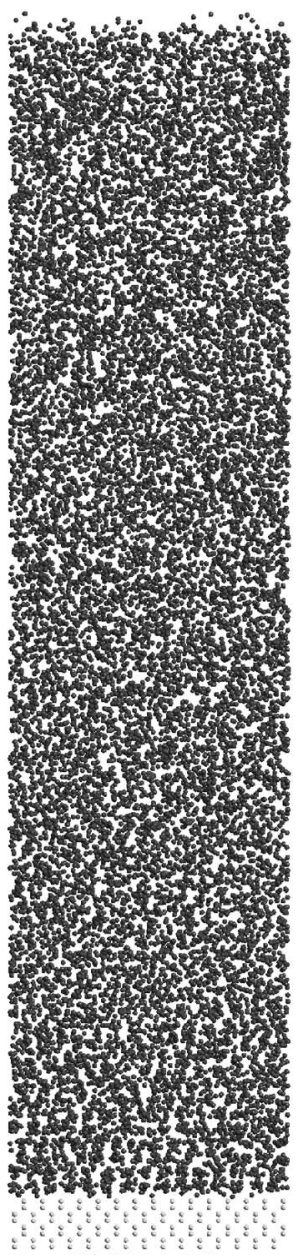

(b) Model B
FIG. 5. Side views of the model structures used for the calculation in Fig. 4, in [110] projection. Dark gray spheres represent Si atoms in the oxide region and light gray spheres represent $\mathrm{Si}$ atoms in the substrate region: (a) Model A: a homogeneous structure and (b) Model B: a gradually disordered structure. to some extent, depending on its formation process. ${ }^{20,24}$ The result obtained indicates that diffusion of atomic oxygen additionally increases the slope of the residual order distribution. In the $\mathrm{O}-400{ }^{\circ} \mathrm{C}$ sample, the residual order persists near the interface but is lost near the surface.

The plasma process for atomic $\mathrm{O}$ generation is also accompanied by generation of UV wavelength photons. Kazor and Boyd performed UV irradiation experiments and suggested an UV-assisted diffusion model involving ionic oxygen formation and space-charge creation due to photoinjection of electrons from $\mathrm{Si}^{3}{ }^{3}$ In that model, drift movement of ionized oxidants due to an electric field caused by the space charge plays an important role. Although the influence of UV irradiation is difficult to assess in the present experiment, it is clear from the present data that atomic $\mathrm{O}$ passing through the $\mathrm{SiO}_{2}$ reacts with the $\mathrm{SiO}_{2}$ network and modifies it.

\section{SUMMARY}

In summary, we present experimental evidence for the recent theoretical predictions on reactions and diffusion of atomic and molecular oxygen in $\mathrm{SiO}_{2}$. Even at a low temperature of $400{ }^{\circ} \mathrm{C}$, atomic oxygen reacts with the $\mathrm{SiO}_{2}$ network near the surface and diffuses toward the interface with changing bond connections. In contrast, molecular oxygen does not react with the $\mathrm{SiO}_{2}$ network at a sufficiently high temperature of $850{ }^{\circ} \mathrm{C}$ for oxidation reaction at the interface. The network modification effect of atomic oxygen found in this work would be useful for relieving the intense stress near the $\mathrm{Si}-\mathrm{SiO}_{2}$ interface or for amorphizing unfavorable polycrystalline region in amorphous oxide films.

\section{ACKNOWLEDGMENTS}

The authors thank M. Takahashi, S. Hamada, and Dr. T. Arikado (Selete, Inc.) for sample preparation. This work was supported by a Grant-in-Aid for COE Research and by the 21COE program "Practical Nano-Chemistry" from MEXT, Japan. The x-ray experiment was performed at the Photon Factory under proposal No. 2004G220.
*Electronic address: tatsumura@toki.waseda.jp

${ }^{1}$ Fundamental Aspects of Silicon Oxidation, edited by Y. J. Chabal (Springer, Berlin, 2001).

${ }^{2}$ J. R. Engstrom, D. J. Bonser, and T. Engel, Surf. Sci. 268, 238 (1992).

${ }^{3}$ A. Kazor and I. W. Boyd, J. Appl. Phys. 75, 227 (1994).

${ }^{4}$ T. Ueno, A. Morioka, S. Chikamura, and Y. Iwasaki, Jpn. J. Appl. Phys., Part 2 39, L327 (2000).

${ }^{5}$ Y. Saito, K. Sekine, M. Hirayama, and T. Ohmi, in The Physics and Chemistry of $\mathrm{SiO}_{2}$ and the $\mathrm{Si}-\mathrm{SiO}_{2}$ Interface-4, edited by H. Z. Massoud, I. J. R. Baumvol, M. Hirose, and E. H. Poindexter (Electrochemical Soc., Pennington, 2000), p. 113.

${ }^{6}$ Y. Saito, K. Sekine, N. Ueda, M. Hirayama, S. Sugawa, and T. Ohmi, in Symp. VLSI Tech. Dig. (2000), p. 176.

${ }^{7}$ B. E. Deal and A. S. Grove, J. Appl. Phys. 36, 3770 (1965).

${ }^{8}$ For a review, M. A. Lamkin, F. L. Riley, and R. J. Fordham, J.
Eur. Ceram. Soc. 10, 347 (1992).

${ }^{9}$ H. C. Lu, T. Gustafsson, E. P. Gusev, and E. Garfunkel, Appl. Phys. Lett. 67, 1742 (1995).

${ }^{10}$ L. Skuja and B. Güttler, Phys. Rev. Lett. 77, 2093 (1996).

${ }^{11}$ F. J. Norton, Nature (London) 191, 701 (1961).

${ }^{12}$ W. G. Perkins and D. R. Begal, J. Chem. Phys. 54, 1683 (1971).

${ }^{13}$ W. Orellana, A. J. R. da Silva, and A. Fazzio, Phys. Rev. Lett. 87, 155901 (2001).

${ }^{14}$ A. Bongiorno and A. Pasquarello, Phys. Rev. Lett. 88, 125901 (2002).

${ }^{15}$ T. Hoshino, M. Hata, S. Neya, Y. Nishioka, T. Watanabe, K. Tatsumura, and I. Ohdomari, Jpn. J. Appl. Phys., Part 1 42, 6535 (2003).

${ }^{16}$ D. R. Hamann, Phys. Rev. Lett. 81, 3447 (1998).

${ }^{17}$ K. O. Ng and D. Vanderbilt, Phys. Rev. B 59, 10132 (1999).

${ }^{18}$ T. Hoshino, M. Hata, S. Neya, Y. Nishioka, T. Watanabe, K. Tat- 
sumura, and I. Ohdomari, Jpn. J. Appl. Phys., Part 1 42, 3560 (2003).

${ }^{19}$ A. Pasquarello, M. S. Hybertsen, and R. Car, Nature (London) 396, 58 (1998).

${ }^{20}$ I. Takahashi, T. Shimura, and J. Harada, J. Phys.: Condens. Matter 5, 6525 (1993).

${ }^{21}$ A. Munkholm, S. Brennan, F. Comin, and L. Ortega, Phys. Rev. Lett. 75, 4254 (1995); T. Shimura, M. Umeno, I. Takahashi, and J. Harada, ibid. 79, 4932 (1997); A. Munkholm, S. Brennan, F. Comin, and L. Ortega, ibid. 79, 4933 (1997).

${ }^{22}$ N. Awaji, Y. Sugita, Y. Hori, and I. Takahashi, Appl. Phys. Lett. 74, 2669 (1999)
${ }^{23}$ K. Tatsumura, T. Watanabe, D. Yamasaki, T. Shimura, M. Umeno, and I. Ohdomari, Phys. Rev. B 69, 085212 (2004).

${ }^{24}$ A. Munkholm and S. Brennan, Phys. Rev. Lett. 93, 036106 (2004).

${ }^{25}$ K. Tatsumura, T. Watanabe, D. Yamasaki, T. Shimura, M. Umeno, and I. Ohdomari, Jpn. J. Appl. Phys., Part 1 42, 7250 (2003).

${ }^{26}$ T. Yamamoto, N. T. Chien, M. Ando, N. Goto, M. Hirayama, and T. Ohmi, Jpn. J. Appl. Phys., Part 1 38, 2082 (1999).

${ }^{27}$ K. Ohtsubo, Y. Saito, M. Hirayama, S. Sugawa, H. Aharoni, and T. Ohmi, IEEE Trans. Plasma Sci. 32, 1747 (2004). 